\title{
GANHO DE PESO E RENDIMENTO DE CARCAÇA DE CORDEIROS MESTIÇOS TEXEL E SANTA INÊS X SRD EM SISTEMA DE MANEJO SEMI-INTENSIV̧O ${ }^{1}$
}

\author{
Weight gain and carcass traits of Texel and Santa Inês crossbred lambs \\ in a semi-intensive husbandry system
}

\author{
Arturo Bernardo Selaive Villarroel ${ }^{2}$, Luiz Evanilton Silva Lima ${ }^{3}$, \\ Sônia Maria Pinheiro de Oliveira ${ }^{2}$, Antônio Amaury Oriá Fernandes ${ }^{4}$
}

\begin{abstract}
RESUMO
Com o trabalho teve-se o objetivo de avaliar o ganho de peso e as características de carcaça de cordeiros mestiços oriundos do cruzamento de carneiros da raça lanada Texel e deslanada Santa Inês com ovelhas deslanadas sem padrão racial definido-SRD. Foram avaliados 33 cordeiros de ambos os sexos, sendo 17 cordeiros mestiços Texel x SRD (8 machos inteiros e 7 fêmeas e 16 mestiços Santa Inês x SRD (11 machos inteiros e 5 fêmeas). Os cordeiros foram desmamados em média com 90 dias de idade e 11,5 kg de peso vivo e mantidos juntos em sistema de manejo semi-intensivo. O ganho de peso foi estimado através de pesagens periódicas a cada 15 dias, do nascimento até os oito meses de idade, período estimado para o abate dos machos. Os cordeiros mestiços Texel foram tosados ao final do experimento para a retirada da lã afim de evitar a influência desta no peso e rendimento da carcaça. A análises dos dados mostraram que os cordeiros mestiços Texel apresentaram maiores pesos corporais ao nascimento e aos oito meses de idade, com ganhos de peso diário significativamente superior $(\mathrm{P}<0,05)$ do desmame ao peso final. O peso final dos cordeiros mestiços Texel foi de $23,70 \pm 3,8 \mathrm{~kg}$ e dos Santa Inês de $20,7 \pm 2,2 \mathrm{~kg}$ com ganhos médios diários, do nascimento até o peso final, de $83 \mathrm{e} 72 \mathrm{~g}$, respectivamente, valores inferiores ao esperado em ambos grupos genéticos. Embora tenha sido obtido um maior peso corporal dos cordeiros mestiços Texel ao abate, não houve influência do genótipo nos rendimentos de carcaça quente e fria dos animais. Conclui-se que a utilização de carneiros da raça Texel em cruzamento com fêmeas deslanadas SRD na região nordeste do Brasil, resulta em maiores ganhos de peso de suas crias, quando comparado com os da raça local Santa Inês, porém, não há efeito do genótipo no rendimento da carcaça dos animais. Desta forma, o uso de reprodutores lanados com ovelhas deslanadas, nas condições em que foram feitas as observações, pode se justificar somente em cruzamento terminal ou industrial e com ovelhas SRD. Maiores estudos devem ser realizados para avaliar o efeito da prdução de lã sobre o desempenho dos animais nas condições de clima do Nordeste.
\end{abstract}

Termos para indexação: Carne ovina, crescimento, cruzamento, ovino.

\begin{abstract}
A research was conducted at the Experimental Field Station Vale do Curu of the Federal University of Ceará to evaluate the growth rate and carcass traits of crossbred lambs born from crioula tropical hair ewes (SRD) mated with Texel and Santa Ines rams. 33 lambs being 17 crossbred Texel X SRD ( eigth entire male lambs and seven female lambs) and 16 crossbred Santa Inês X SRD (11 entire male lambs and five female lambs) were studied. Lambs were weaned at 90 days old and were raised in a semi-intensive system production. Crossbred Texel lambs were sheared before slaugther. Growth rate was studied by measuring the live bodyweight each 15 days from born to eigth months old, age estimated as good to slaughter the male lambs. The weigth and carcass yield were evaluated. Data analyses show that the crossbred Texel lambs have better growing performance and higher daily weigth gain than Santa Inês crossbred lambs, but significant differences of sire breed were not detected in the hot and cold carcass yield. It was concluded that crossbreeding of crioula tropical hair ewes with Texel rams may be beneficial for growth performance of lambs in a industrial breeding programme. More studies are necessary to evaluate the effect of wool on sheep raising on tropical condition.
\end{abstract}

Index terms: Cross-breeding, ovine, carcass quality, lambs.

(Recebido para publicação em 25 de junho de 2004 e aprovado em 6 de junho de 2006)

INTRODUÇ̃̃o

A produção de carne ovina é uma alternativa viável e econômica para incrementar o desenvolvimento rural no Brasil, inclusive dentro de programas de desenvolvimento da agricultura familiar (SOUZA NETO, 1987). Neste sentido, o borrego com aproximadamente $28-30 \mathrm{~kg}$ de peso vivo é a categoria de maior aceitabilidade no mercado consumidor e de melhores características de carcaça (SIQUEIRA, 1999).

Nos últimoa anos, a demanda de carne ovina tem aumentado significativamente na região Nordeste, fazendo parte da culinária regional e, embora seja a maior região de população ovina no País, existe um defícit de oferta compensado pela importação de carne de outros países, principalmente Uruguay e Argentina. Tendo em vista que

\footnotetext{
${ }_{1}$ Parte da Dissertação de Mestrado apresentada ao DZ/CCA/UFC pelo segundo autor. Apoio FUNCAP.

2 Professor do Departamento de Zootecnia da Universidade Federal do Ceará/UFC - Av. Pintor Antônio Bandeira, 1751 - Apto 801 - $60.183-301$ Fortaleza, CE - Selaive@ufc.br

${ }^{3}$ Centro Vocacional de Rusas - CENTEC - Av. José Sobreira da Cruz, 323 -Bairro Centro - 60.040-260 - Missão Velha, CE.

${ }^{4}$ Professor da Faculdade de Veterinária da UECE - Rua General Castelo Branco, 070 - Bairro Cidade dos Funcionários - 60.822-040.
} 
a maioria dos ovinos no Nordeste são animais sem padrão racial definido (SRD) e que apresentam reduzido crescimento e pouco peso corporal ao abate, com carcaças de reduzido rendimento e deficiente conformação e qualidade (SELAIVE-VILLARROEL \& SOUZA JÚNIOR, 2005), faz-se necessário estudos visando a obtenção de genótipos de maior potencialidade para carne.

O emprego do cruzamento, com a utilização de raças exóticas especializadas para produção de carne como linha paterna e as raças locais como linha materna, constitui uma ferramenta para obtenção de produtos de maior crescimento e melhor qualidade comercial de carcaça e de carne (CARDELLINO, 1989). Segundo Sainz (1996), a composição e a qualidade da carcaça são características importantaes para estabelecer cruzamentos de raças paternas especializadas para carne com matrizes adaptadas à região. Assim, o conhecimento dos efeitos de diferentes raças paternas sobre a progênie permite orientar a produção comercial de carne ovina. Entre as raças especializadas para corte, a Texel é muito comum no Sul do País, pela sua rusticidade, aliada a sua precocidade e produção de carcaça considerada como de excelente qualidade por alguns autores (FURUSCHO-GARCIA et al., 2004).

Diversas pesquisas têm sido desenvolvidas na região visando o ganho de peso de cordeiros em crescimento, porém, é pouca a informação avaliando o impacto do cruzamento de reprodutores de raças especializadas para carne em acasalamento com matrizes nativas do nordeste brasileiro (BARROS et al., 1999). A quantificação da produtividade e eficiência biológica das crias mestiças permite fornecer informações básicas para a melhoria da produção de carne ovina na região. Levandose em consideração estes aspectos, avaliou-se o crescimento e as características de carcaça de cordeiros mestiços oriundos do cruzamento de matrizes deslanadas sem raça definida (SRD) com reprodutores de raça lanada Texel, tendo como testemunha cordeiros de reprodutores da raça local Santa Inês, com o objetivo de determinar alternativas adequadas de cruzamento nas condições de criação do semi-árido nordestino.

\section{MATERIAL E MÉTODOS}

O trabalho foi realizado na Fazenda Experimental Vale do Curu pertencente à Universidade Federal do Ceará, Pentecoste-CE. Foram avaliados 33 cordeiros de ambos os sexos, sendo 17 cordeiros mestiços Texel X SRD (8 machos inteiros e 7 fêmeas) e 16 cordeiros Santa Inês X SRD (11 machos inteiros e 5 fêmeas), mantidos em sistema semiintensivo de produção. Após o nascimento, os cordeiros ficaram com as mães até o desmame, com aproximadamente 90 dias de idade. Após o desmame as crias foram mantidas numa área de 18,0 ha de pastagem nativa melhorada (caatinga raleada) durante o dia e confinados no final da tarde, quando receberam uma suplementação alimentar de concentrado comercial com $20 \%$ de proteína, à base de farelo de trigo, soja, milho, leucena e ureia, além do sal mineral, em quantidades crescentes de 200 gramas por cabeça/dia no primeiro mês (de 3 ate os 4 meses de idade), 300 gramas ate os seis meses de idade e, após, 400 gramas atê o final do experimento. Os cordeiros mestiços Texel foram tosados antes do abate para a retirada da lã afim de evitar a influência desta no peso e rendimento da carcaça.

O desempenho dos cordeiros foi avaliado através de pesagens periódicas a cada 15 dias, do nascimento até os oito (8) meses de idade. Os cordeiros machos foram abatidos inteiros (não castrados), após jejum e dieta hídrica de 24 h, com idade média de 240 dias. Após abate e evisceraçào, foi avaliado o rendimento da carcaça quente (RCQ), calculada pela relação entre PCQ/PA x 100 e as carcaças resfriadas por 24 horas, em câmara fria a $2^{\circ} \mathrm{C}$ com 95\% de umidade, sendo novamente pesadas para determinação do rendimento da carcaça fria (RCF) calculada pela relação PCF/PCQ x 100, segundo a metodologia descrita por Osório et al. (1998).

Os dados de peso corporal ao nascimento (PN), desmame ( $\mathrm{PD}=90$ dias de idade), peso final $(\mathrm{PF}=240 \mathrm{de}$ idade), pesos intermediários (P1, P2, P3 ..) e peso de abate (PA=PF-24 horas jejum), bem como os ganhos de peso diário (GPD) e ganho de peso total (GPT) e os dados de peso da carcaça quente e fria e seus rendimentos quente e fria foram analisados pelo procedimento GLM (General Linear Models) do programa "Statistical Analysis System" (SAS INSTITUTE, 1999) e as comparações de médias feitas pelo teste de Tukey, considerando o efeito genético e do sexo dos animais como fonte de variação e o efeito do peso da ovelha à parição como covariável. As análises para as características de desenvolvimento corporal foram feitas de acordo com o seguinte modelo:

em que:

$$
Y_{i j k}=m+G_{i} S_{j}+b_{1}\left(P_{i j k}-P\right)+e_{i j k},
$$

Yijk = peso e ganho de peso das características do animal $\mathrm{k}$, do grupo genético $\mathrm{i}$, e do sexo $\mathrm{j}$;

$\mathrm{m}=$ média geral ;

$\mathrm{G}_{\mathrm{i}}=$ efeito do grupo genético $(\mathrm{i}=1$ e 2$)$;

$\mathrm{S}_{\mathrm{j}}=$ efeito do sexo da cria $\mathrm{j}$ (1 macho inteiro e 2 fêmea);

$\mathrm{b}_{1}=$ coeficiente de regressão linear de $\mathrm{P}_{\mathrm{ijk}}$ em relação ao peso da ovelha à parição ;

$\mathrm{P}_{\mathrm{ij}}=$ peso da ovelha à parição $\mathrm{k}$, na observação 1 , do

Ciênc. agrotec., Lavras, v. 30, n. 5, p. 971-976, set./out., 2006 
genótipo i e sexo $\mathrm{j}$;

$\mathrm{P}=$ média do peso da ovelha à parição ;

$\mathrm{e}_{\mathrm{ijkl}}=$ erro aleatório associado a cada observação.

Os efeitos das interações entre os efeitos fixos foram testados para todas as características estudadas e como não apresentaram resultados significativos, foram retiradas do modelo. Foram feitos os ajustamentos dos pesos para as idades de 90 e 240 dias e estimados os ganhos de peso médios diários.

\section{RESULTADOS E DISCUSSÃO}

A análise dos pesos corporais mostrou que houve efeito significativo $(\mathrm{P}<0,05)$ do genótipo sobre o PN e o PF dos cordeiros, porém não foi observada diferença significativa no PD, conforme se observa na Tabela 1 . $\mathrm{O}$ sexo da cria e o peso da ovelha à parição não influenciaram significativamente nenhum peso corporal do cordeiro, observando-se o mesmo com a interação sexo x genótipo.

$\mathrm{O}$ peso médio ao nascer das crias oriundas do cruzamento Santa Inês x SRD foi de 2,87 $\pm 0,47 \mathrm{~kg}$, enquanto que o dos mestiços Texel x SRD foi de 3,25 $\pm 0,63 \mathrm{~kg}$, havendo diferença significativa $(\mathrm{P}<0,05)$ entre ambos os grupos. O maior peso observado nos cordeiros mestiços Texel está de acordo com o relatado na literatura nacional para cruzamento de reprodutores lanados com ovelhas deslanadas SRD no Nordeste Brasileiro, quando os cordeiros mestiços lanados apresentam peso ao nascimento superior aos deslanados (BARROS et al., 1999; FURUSHO-GARCIA et al., 2004; MACHADO et al., 1999). Entretanto, o peso ao nascimento observado neste trabalho é inferior ao relatado na literatura, em que são descritos valores de 3,22 kg e 3,82 kg para dos cordeiros mestiços Santa Inês e Texel, respectivamente em parições em confinamento (MACHADO et al., 1999).

Já no desmame, com média de 90 dias de idade, não observou-se diferença significativa $(\mathrm{P}>0,05)$ no peso corporal entre ambos grupos de cordeiros. $\mathrm{O}$ peso médio ao desmame dos mestiços Santa Inês foi de 11,37 $\pm 1,43 \mathrm{~kg}$, enquanto nos mestiços Texel foi de $11,68 \pm 2,87 \mathrm{~kg}$, com ganhos de peso real de $8,48 \mathrm{~kg}$ e $8,43 \mathrm{~kg}$, respectivamente. A não diferença de peso ao desmame entre os cordeiros de ambos grupos genéticos deve-se provavelmente à similar base genética materna, com semelhante produção de leite das ovelhas, permanecendo os cordeiros com às mães nas mesmas condições de alimentação. Considerando que a produção de leite da ovelha influi diretamente no peso ao desmame (MOTTA et al., 2000) é possível que o leite produzido pelas ovelhas tenha sido insuficiente para que os cordeiros mestiços Texel pudessem expressar seu maior potencial genético para crescimento como raça mais especializada para corte, podendo esta ser a explicação da ausência de significância no peso corporal ao desmame entre os cordeiros de ambos grupos genéticos.

No período pós-desmame, onde não há influência da mãe, o peso final dos cordeiros com 240 dias de idade, foi significativamente superior nos mestiços Texel que os do Santa Inês, com valores de 23,7 $\pm 3,8 \mathrm{~kg}$ e $20,7 \pm 2,2 \mathrm{~kg}$, respectivamente, sendo o ganho real de 17,35 e $20,49 \mathrm{~kg}$, para os mestiços Santa Inês e Texel, respectivamente. Esta diferença é explicada pelo maior potencial para ganho de peso da raça Texel, por ser mais selecionada para carne e conseqüentemente apresentar maior velocidade de crescimento e maior conversão alimentar que a Santa Inês, raça considerada mais rústica conforme constatado por Barros et al. (1999). Entretanto, os pesos atingidos pelos animais foram muito abaixo do esperado para cordeiros mestiços e inferiores aos descritos na literatura (SELAIVEVILLARROEL \& SOUZA JÚNIOR, 2005). Além do reduzido crescimento e ganho de peso que caracteriza aos ovinos da região Nordeste do País, o baixo peso dos animais mestiços observados neste trabalho pode ser explicado pelo fato das ovelhas utilizadas no cruzamento terem sido animais sem padrões raciais definidos, o que significa que não tiveram algum grau de seleção, além da seleção natural, o que se repercute nas progênies com crescimento menor do que ocorre quando se cruzam animais de raças definidas.

TABELA 1 - Médias e desvios padrão do peso corporal (kg) de cordeiros ao nascimento, ao desmame e peso final, segundo o genótipo.

\begin{tabular}{lcccc}
\hline \multicolumn{1}{c}{ Genótipo } & $\begin{array}{c}\mathbf{N}^{\mathbf{a}} \text { de } \\
\text { observações }\end{array}$ & $\begin{array}{c}\text { Peso ao nascimento } \\
(\mathbf{P N})\end{array}$ & $\begin{array}{c}\text { Peso ao desmame } \\
(\mathbf{P D})\end{array}$ & $\begin{array}{c}\text { Peso final } \\
(\mathbf{P F})\end{array}$ \\
\hline Santa Inês x SRD & 16 & $2,87 \pm 0,47^{\mathrm{b}}$ & $11,37 \pm 1,43^{\mathrm{a}}$ & $20,70 \pm 2,18^{\mathrm{b}}$ \\
Texel x SRD & 17 & $3,25 \pm 0,63^{\mathrm{a}}$ & $11,68 \pm 2,87^{\mathrm{a}}$ & $23,70 \pm 3,81^{\mathrm{a}}$ \\
\hline
\end{tabular}

Letras diferentes na mesma coluna indicam diferença significativa ao nível de $5 \%$. 
Como era esperado, pelos resultados obtidos nos diferentes pesos corporais dos animais, não houve diferenças significativas $(P>0,05)$ no ganho de peso do nascimento ao desmame entre os cordeiros mestiços Texel e Santa Inês em relação aos GPD e GPT. Entretanto, foram significativas as diferenças entre os GPD e GPT do desmame ao peso final, com 240 dias de idade, da mesma forma que do nascimento ao peso final $(\mathrm{P}<0,05)$. Do nascimento até o desmame, o GPD foi de 85 e 89 gramas e, do desmame ao peso final, foi de 63 e 80 gramas, respectivamente para os mestiços Santa Inês e Texel. O ganho médio diário total, do nascimento ao peso final, foi de 72 e 83 gramas, respectivamente, conforme se observa na Tabela 2.

A semelhança do peso corporal, os valores obtidos para ganho médio diário do desmame até os 240 dias de vida, são considerados muito reduzidos, porém, nas referidas médias estão incluídos valores referentes a períodos de perda de peso, nos quais os animais permaneceram na pastagem nativa sob condições climáticas adversas na época seca ou em chuvas excessivas.

$\mathrm{O}$ crescimento dos cordeiros mestiços Santa Inês e Texel obedeceu a um padrão de curva semelhante, conforme é mostrado no Figura 1.

TABELA 2 - Médias e desvio padrão dos ganhos de peso diário (GPD) e total (GPT) de cordeiros, segundo o genótipo e o sexo.

\begin{tabular}{lcccccc}
\hline & \multicolumn{2}{c}{$\begin{array}{c}\text { Ganho de Peso } \\
\text { Nascimento-Desmame }\end{array}$} & \multicolumn{2}{c}{$\begin{array}{c}\text { Ganho de peso } \\
\text { Desmame-Peso final }\end{array}$} & \multicolumn{2}{c}{$\begin{array}{c}\text { Ganho Total } \\
\text { Nasc.-Peso final }\end{array}$} \\
\cline { 2 - 7 } & GPD (g/dia) & GPT (kg) & GPD (g/dia) & GPT (kg) & GPD (g/dia) & GPT (kg) \\
\hline Genótipo: & & & & & & \\
Santa Inês x SRD & $85^{\mathrm{a}}$ & $8,48^{\mathrm{a}}$ & $63^{\mathrm{a}}$ & $9,38^{\mathrm{a}}$ & $72^{\mathrm{a}}$ & $17,85^{\mathrm{a}}$ \\
Texel x SRD & $89^{\mathrm{a}}$ & $8,43^{\mathrm{a}}$ & $80^{\mathrm{b}}$ & $12,09^{\mathrm{b}}$ & $83^{\mathrm{b}}$ & $20,49^{\mathrm{b}}$ \\
Sexo & & & & & & \\
Macho & $90^{\mathrm{a}}$ & $8,54^{\mathrm{a}}$ & $72^{\mathrm{a}}$ & $10,73^{\mathrm{a}}$ & $79^{\mathrm{a}}$ & $19,27^{\mathrm{a}}$ \\
Fêmea & $83^{\mathrm{a}}$ & $8,34^{\mathrm{a}}$ & $72^{\mathrm{a}}$ & $10,76^{\mathrm{a}}$ & $76^{\mathrm{a}}$ & $19,08^{\mathrm{a}}$ \\
\hline
\end{tabular}

Letras minúsculas diferentes na mesma coluna indicam diferença significativa ao nível de $5 \%(\mathrm{P}<0,05)$, pelo teste de Tukey.

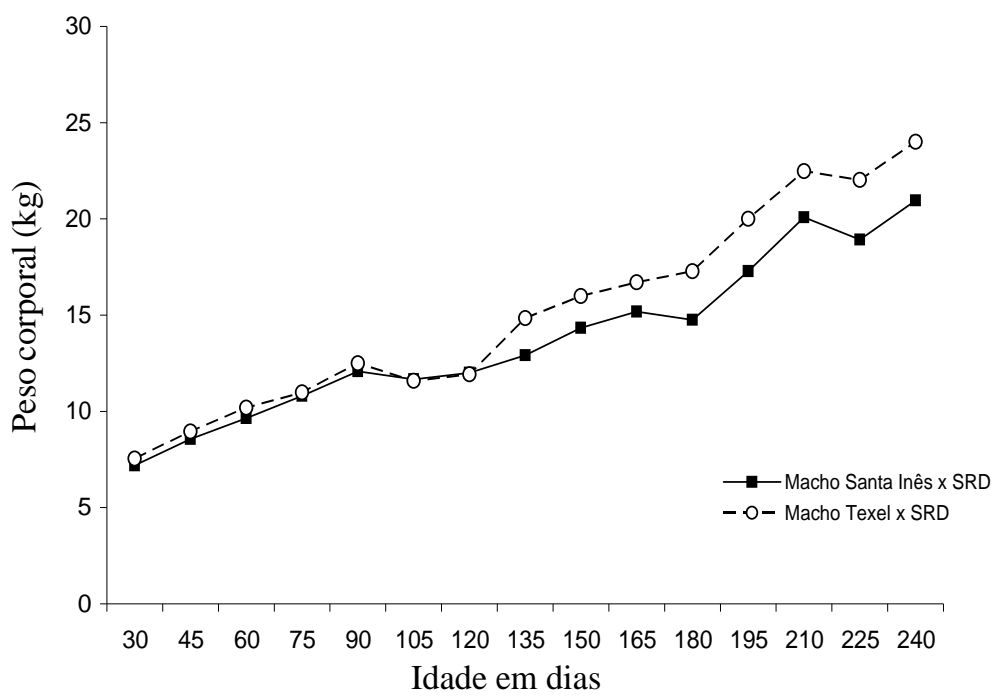

FIGURA 1 - Curva de crescimento de cordeiros mestiços Santa Inês x SRD e Texel x SRD do nascimento até os 240 dias de idade.

Ciênc. agrotec., Lavras, v. 30, n. 5, p. 971-976, set./out., 2006 
Os resultados obtidos neste trabalho, confirmam o relatado na literatura (CARDELLINO, 1989) no sentido do que animais filhos de reprodutores de raças especializadas para corte apresentam maior velocidade de crescimento que aqueles de raças mais rústicas.

Em todo o período experimental, o ganho de peso dos cordeiros de ambos grupos genéticos foi inferior ao esperado conforme relatado na literatura nacional. Assim, Barros et al. (1999), em cordeiros mantidos em confinamento oriundos do cruzamento de reprodutores das raças lanadas Hampshire Down, Suffolk e Ile de France com ovelhas SRD, descrevem médias de ganho de peso pós-desmame de 160 a 180 gramos diários. Por outro lado, Furusho et al. (1995), em cordeiros mestiços oriundos de raças puras Texel e Santa Inês, mantidos em confinamento, encontraram ganhos de peso médio de 274,0 g/dia do nascimento até o desmame e de 191,8 g/dia do desmame até os 180 dias de idade. Embora as diferenças encontradas devam-se, entre outras, às diferenças no sistema de produção empregado e a idade de abate dos animais, os resultados mostraram que o crescimento de cordeiros oriundos do cruzamento entre raças puras é superior ao observado quando uma das raças não apresenta padrão racial definido e, portanto, nunca teve algum grau de seleção.

O sexo da cria não teve influência significativa entre os cordeiros nos diferentes pesos corporais estudados. Nos cordeiros machos, o PN foi de $3,08 \pm 0,50 \mathrm{~kg}$, o PD foi de $11,62 \pm 1,86 \mathrm{~kg}$ e o $P F$ de $22,35 \pm 3,65 \mathrm{~kg}$, enquanto que nas fêmeas foram: $\mathrm{PN}=3,03 \pm 0,70 \mathrm{~kg} P D=11,37 \pm 3,79 \mathrm{~kg}$; e $\mathrm{PF}=22,10 \pm 3,24 \mathrm{~kg}$. Era de se esperar que os machos apresentassem peso superior ao das fêmeas, uma vez que os animais do sexo masculino tendem a apresentar uma maior velocidade no ganho de peso que as fêmeas, conforme demonstrado nos trabalhos relatados na literatura (LLOYD et al., 1983; SIQUEIRA et al., 2001).

Em relação à carcaça, não foram observadas diferenças $(\mathrm{P}>0,05)$ no peso e rendimento da carcaça quente e fria entre os grupos de animais abatidos com oito meses de idade. Os pesos de carcaça quente foram de 9,81 $\mathrm{kg}$ e $8,41 \mathrm{~kg}$ e os rendimentos foram $40,4 \%$, e $39,1 \%$, respectivamente para os cordeiros mestiços Texel e Santa Inês, conforme se observa na tabela 3.
O conhecimento do rendimento é fundamental para estimar o valor comercial da carcaça, pois é uma característica diretamente relacionada à produção e comercialização de carne e expressa a porcentagem de peso da carcaça obtida em relação a um peso vivo determinado, podendo variar em função de fatores intrínsecos relacionados ao próprio animal (genótipo, sexo, peso, idade) e/ou extrínsecos (alimentação, manejo, tipo de jejum) do animal (SILVA SOBRINHO, 2001). O maior rendimento de carcaça esperado nos cordeiros mestiços Texel sobre os Santa Inês, pelo fato de ser uma raça mais especializada para carne, não foi observado. Isto provavelmente devese ao baixo vigor híbrido que possui esta característica (DHANDA et al., 2003) ou a alimentação não ter sido suficiente para atender as maiores exigências nutricionais da raça Texel. Inexistência de diferenças significativas entre grupos mestiços foi observada também, em ovinos, por Siqueira et al. (2001).

Por outro lado, os resultados de rendimento de carcaça nos cordeiros mestiços Santa Inês foram inferiores aos observados pelos autores em trabalhos anteriores em cordeiros mestiços Santa Inês e Somalis abatidos com seis meses de idade (SELAIVE-VILLARROEL \& SOUZA JÚNIOR, 2005), bem como os de Garcia et al. (2000) em cordeiros cruzas Texel x Santa Inês.. Os resultados obtidos mostraram que o rendimento da carcaça de cordeiros oriundos de ovelhas sem raça definida constitui um ponto de estrangualmento na produção de carne ovina no nordeste.

Um aspecto importante a ser considerado neste trabalho, foi a presença de lã nos cordeiros mestiços de Texel, fato já esperado e considerado negativo para as condições de criação no Nordeste. Foram observadas quantidades de lã que variaram de $0,30 \mathrm{~kg} \mathrm{a} 0,80 \mathrm{~kg}$ por animal, o que comercialmente não é viável nas condições do Nordeste do Brasil, além da competição deste produto por nutrientes que poderiam ser canalizados para o desenvolvimento de carcaça e outros tecidos comestíveis. Portanto, o uso de reprodutores Texel no nordeste poderia ser utilizado apenas para cruzamento industrial e com ovelhas SRD.

TABELA 3 - Médias e desvio padrão de peso (PCQ) e rendimento (RCQ) de carcaça quente de cordeiros mestiços Santa Inês e Texel.

\begin{tabular}{lccc}
\hline \multicolumn{1}{c}{ Genótipo } & $\mathbf{N}^{\mathbf{0}}$ observações & PCQ $(\mathbf{k g})$ & RCQ $(\%)$ \\
\hline Santa Inês x SRD & 11 & $8,41 \pm 0.97^{\mathrm{a}}$ & $39,91 \pm 1.64^{\mathrm{a}}$ \\
Texel x SRD & 8 & $9,81 \pm 2.34^{\mathrm{a}}$ & $40,39 \pm 3.22^{\mathrm{a}}$ \\
\hline
\end{tabular}

Letras minúsculas semelhantes na mesma coluna indicam que não há diferença significativa $(P>0,05)$, pelo teste de Tukey. 


\section{CONCLUSÕES}

A utilização de carneiros lanados da raça Texel sobre fêmeas deslanadas SRD resulta em maiores ganho de peso de suas crias, quando comparado com as da raça local Santa Inês, porém, não há efeito significativo no rendimento da carcaça. $\mathrm{O}$ emprego de reprodutores Texel no Nordeste pode ser utilizado para cruzamento terminal, e com ovelhas SRD. Maiores estudos são necessários ao respeito, incluindo outras raças similares.

\section{REFERÊNCIAS BIBLIOGRÁFICAS}

BARROS, N. N.; FIGUEIREDO, E. A. P.; BARBIERE, M. Efeito do genótipo e da alimentação no desempenho de borregos de cruzamento industrial em confinamento. Revista Científica de Produção Animal, Brasília, v. 1, n. 1, p. 59-67, 1999.

CARDELlinO, R. A. Producción de carne ovina basada en cruzamientos. In: Selección de temas agropecuários. Montevideo: Hemisferio Sur, 1989. 520 p.

DHANDA, J. S.; TAYLOR, D. G.; MURRAY, P. J. Growth, carcass and meat quality parameters of male goats: part 1: effects of genotype and liveweight at slaughter. Small Ruminant Research, [S.1.], v. 50, p. 57-66, 2003.

FURUSHO-GARCIA, I. F.; PEREZ, J. R. O.; BONAGURIO, S.; ASSIS, R. de M.; PEDREIRA, B. C.; SOUZA, X. R. Desempenho de cordeiros Santa Inês puros e cruzas Santa Inês com Texel, Ile de France e Bergamácia. Revista Brasileira de Zootecnia, Viçosa, v. 33, n. 6, p. 1591-1603, 2004.

GARCIA, I. F. F.; PEREZ, J. R. O.; OLIVEIRA, M. V. de. Características de carcaça de cordeiros Texel X Bergamásia, Texel X Santa Inês e Santa Inês Puros, terminados em confinamento, com casca de café como parte da dieta. Revista Brasileira de Zootecnia, Viçosa, v. 29, n. 1, p. 253-260, 2000.

LLOYD, W. R.; SLYTER, A. L.; COSTELLO, W. J. Effect of breed, sex and final weigth on feedlot performance, carcass characteristics and meat palatability of lambs. Journal of Animal Science, Champaign, v. 51, p. 316-320, 1983.

MACHADO, R.; SIMPLICIO, A. A.; BARBIERI, M. E. Acasalamento entre ovelhas deslanadas e reprodutores especializados para corte: desempenho produtivo até a desmama. Revista Brasileira de Zootecnia, Viçosa, v. 28, n. 4, p. 706-712, 1999.
MOTTA, O. S.; PIRES, C. C.; SILVA, J. H. da; ROSA, G. T. da; FULBER, M.; GARCIA, A. G. Produção de leite de ovelhas e suas correlações com o ganho de peso dos cordeiros. Revista Brasileira de Zootecnia, Viçosa, v. 29, n. 1, p. 273-279, 2000.

OSÓRIO, J. C. S.; OSÖRIO, M. T. M.; JARDIM, P. O. Métodos para avaliação da produção da carne ovina: in vivo na carcaça e na carne. Pelotas: UFPEL, 1998. 99 p.

SAINZ, R. D. Qualidade das carcaças e da carne ovina e caprina. In: REUNIÃO ANUAL DA SOCIEDADE BRASILEIRA DE ZOOTECNIA, 33., 1996, Fortaleza, CE. Anais... Fortaleza: Sociedade Brasileira de Zootecnia, 1996. p. 3-14.

SAS INSTITUTE. The SAS system for windows version 8.0. Carry, 1999. 3 CD-ROM.

SELAIVE-VILLARROEL, A. B.; SOUSA JUNIOR, F. A. de. Crescimento e caracteristicas de carcaça de cordeiros mestiços Santa Inês e Somalis x SRD em regime semiintensivo de criação. Revista Ciência e Agrotecnologia, Lavras, v. 29, n. 5, 2005.

SIQUEIRA, E. R. Confinamento em ovinos. In: SIMPÓSIO PAULISTA DE OVINOCULTURA E ENCONTRO INTERNACIONAL DE OVINOCULTURA, 5., 1999, Botucatu. Anais... Botucatu: UNESP; CATI; IZ; ASPACO, 1999. p. 52-59.

SIQUEIRA, E. R.; SIMÕES, C. D.; FERNANDES, S. Efeito do sexo e do peso ao abate sobre a produção de carne de cordeiro: I. velocidade de crescimento, caracteres quantitativos da carcaça, $\mathrm{pH}$ da carne e resultado econômico. Revista Brasileira de Zootecnia, Viçosa, v. 30, n. 3, p. 844-848, 2001.

SILVA SOBRINHO, A. G. Aspectos quantitativos e qualitativos da produção de carne ovina. In: PRODUÇÃO ANIMAL NA VISÃO DOS BRASILEIROS, 2001, Piracicaba. Anais... Piracicaba: FEALQ, 2001. p. 425-446.

SOUZA NETO, J. de. Demanda potencial de carne de caprinos e ovinos e perspectiva da oferta 1985/1990. Sobral: Embrapa-CNPC, 1987. 16 p. (Documentos, 2).

Ciênc. agrotec., Lavras, v. 30, n. 5, p. 971-976, set./out., 2006 